\title{
Histopathologic detection of clinically unsuspected cerebral metastatic choriocarcinoma: a case report
}

\begin{abstract}
Unsuspected cerebral metastatic choriocarcinoma is very rare but well documented in medical literature. We present a histopathology case report of clinically unsuspected cerebral metastatic choriocarcinoma in a 41 year old non-hypertensive female. Histopathologic features of the tumor, histopathologic differential diagnosis, role of immunochemistry and importance of other supportive laboratory investigations are discussed.
\end{abstract}

Keywords: intracerebral hemorrhage, ICH, choriocarcinoma
Volume 3 Issue 2 - 2016

Munaf Desai, Farheen Ala

Department of Histopathology and Cytopathology, UAE

Correspondence: Munaf Desai, Department of Pathology, Al Qassimi Hospital, Sharjah, UAE, Tel 00971503622402, Email munafdesai@yahoo.com

Received: October 25, 2015 | Published: November 30, 2016

\section{Introduction}

Intracerebral hemorrhage can occur due to primary and metastatic intracranial neoplasm. In half of the cases it may be the first manifestation of the tumor. It is also known that metastatic tumors bleed more than the primary CNS tumor. ${ }^{1}$ Metastatic choriocarcinoma is a rare but an important cause of intra cerebral hemorrhage in female patients in reproductive age group. Choriocarcinoma (gestational/ non-gestational) is a malignant trophoblastic disease. Incidence of gestational choriocarcinoma is 1 in 50,000 term pregnancy and 1 in 30 hydatidifirm moles. The risk of gestational choriocarcinoma is rare in females under 20years and it is significantly increased after 40year. ${ }^{2}$ Non-gestational choriocarcinoma is very rare and occurs in the overies of prepubertal girls and the testes in males. ${ }^{3}$ The usual clinical presentation of the choriocarcinoma is abnormal uterine hemorrhage after abortion or hydatidiform mole. But, rarely, intracerebral hemorrhage can be the first manifestation of it.

\section{Case report}

A specimen labeled as left frontoparietal hematoma-? cavernoma from a 41year old non-hypertensive female patient on ventilator was received for histopathologic examination. The patient was transferred from outside hospital to neurosurgical department of our hospital for craniotomy and evacuation of intracerebral hematoma. The clinoradiologic diagnosis was spontaneous intracerebral hematoma with intraventricular extension with normal ventricle, probably due to cavernoma. The microscopic examination of the specimen revealed extensive hemorrhage with peripherally placed mono-nucleated and multinucleated atypical cells. The mononucleotide cells were medium to large size with abundant cytoplasm and central large hyperchromatic nuclei. The multinucleated cells were large with abundant eosinophilic cytoplasm and hyperchromatic multinucleated bizarre nuclei. They were considered as cytotrophoblasts and syncytiotrophoblasts respectively (Figure 1-4). Immunohistochemistry showed that the atypical cells were CKAE1AE3+, HCG+, GFAP-, CD31- and Vimentin-(Figure 5-10). A thin rim of GFAP positive glial tissue was seen in one fragment. Based on these findings, a diagnosis of metastatic choriocarcinoma was made. The patient survived the surgery and started recovering neurologically, but chose to go back to her own country for further management. So, we could not get any details of her obstetric history or previous medical illness.

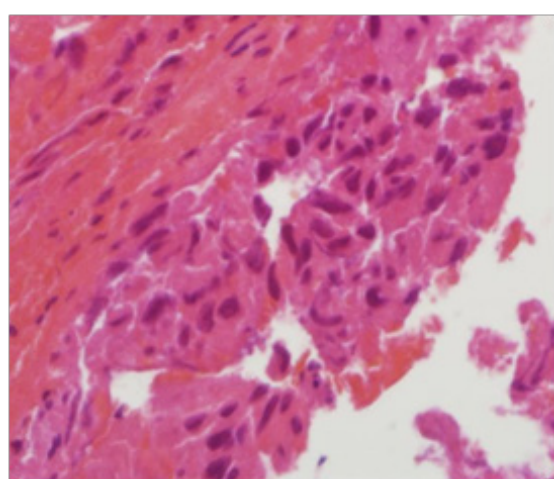

Figure I Cytotrophoblasts.

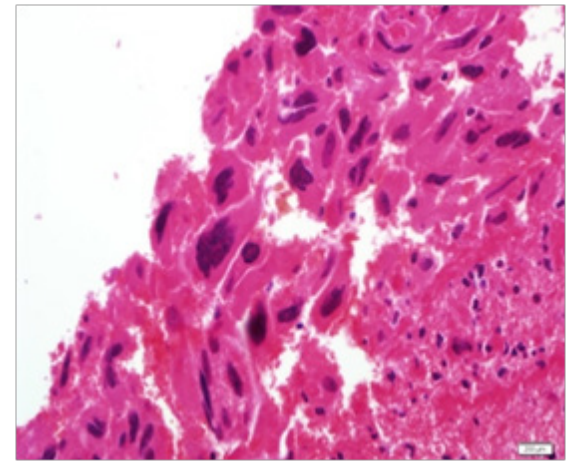

Figure 2 Syncytiotophoblasts.

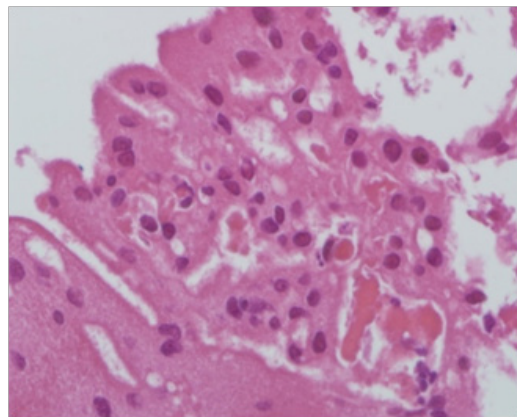

Figure 3 Cytotrophoblasts. 


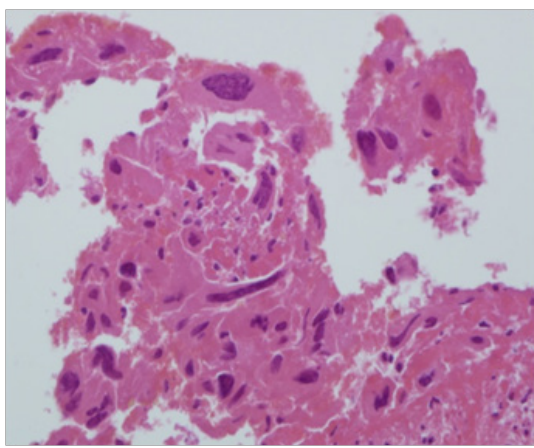

Figure 4 Syncytiotophoblasts.

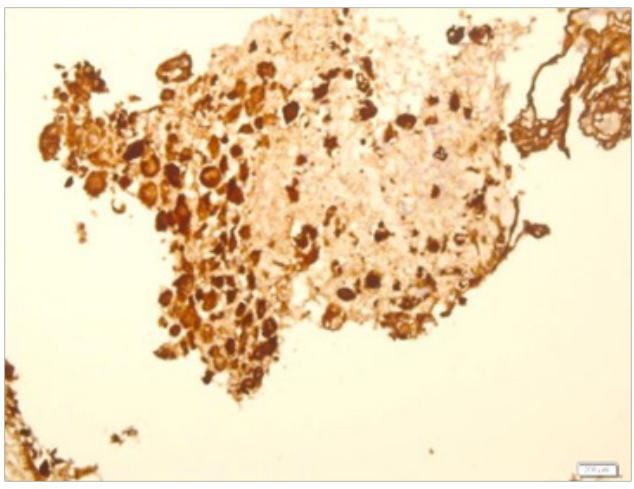

Figure 5 CKAEIAE3.

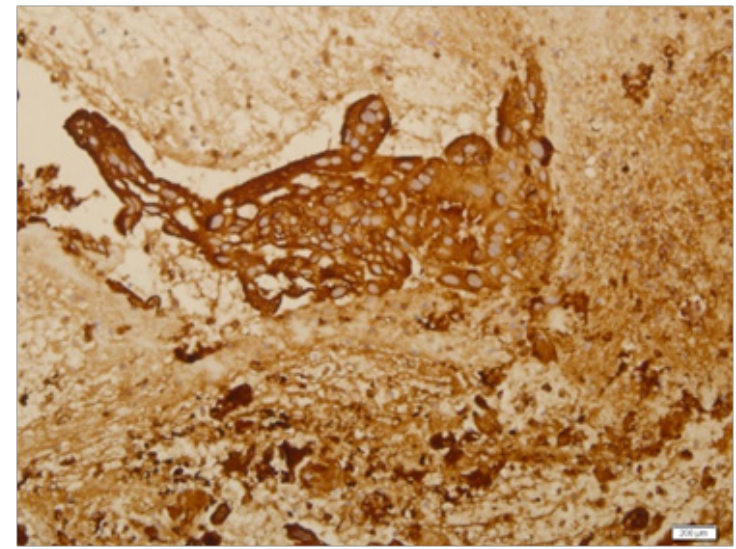

Figure 6 CKAEIAE3.

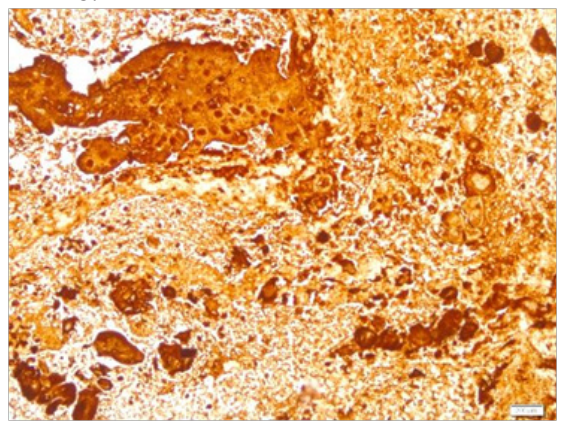

Figure 7 HCG.

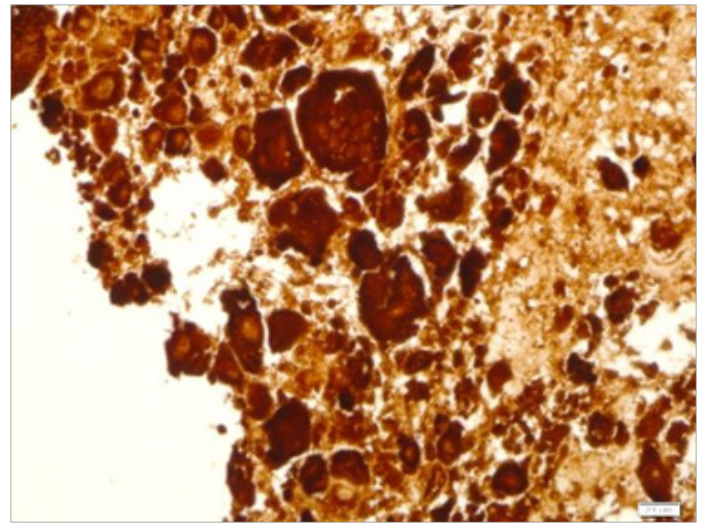

Figure 8 HCG.

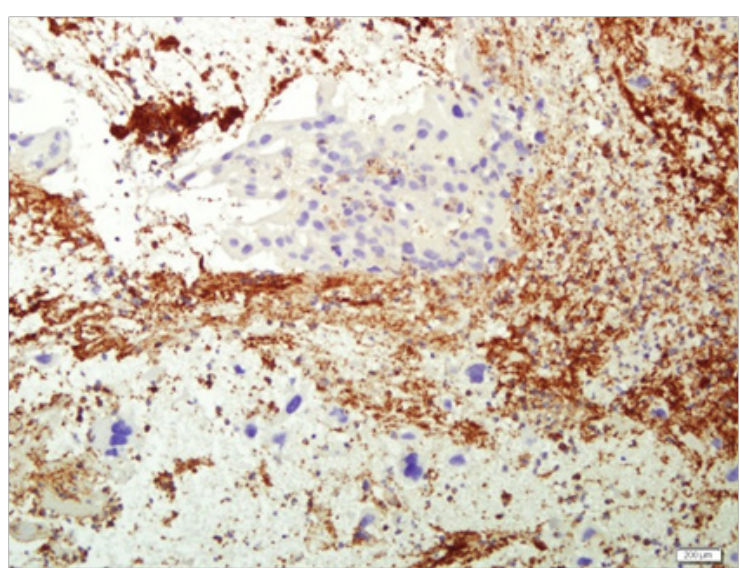

Figure $9 \mathrm{CD} 3 \mathrm{I}$.

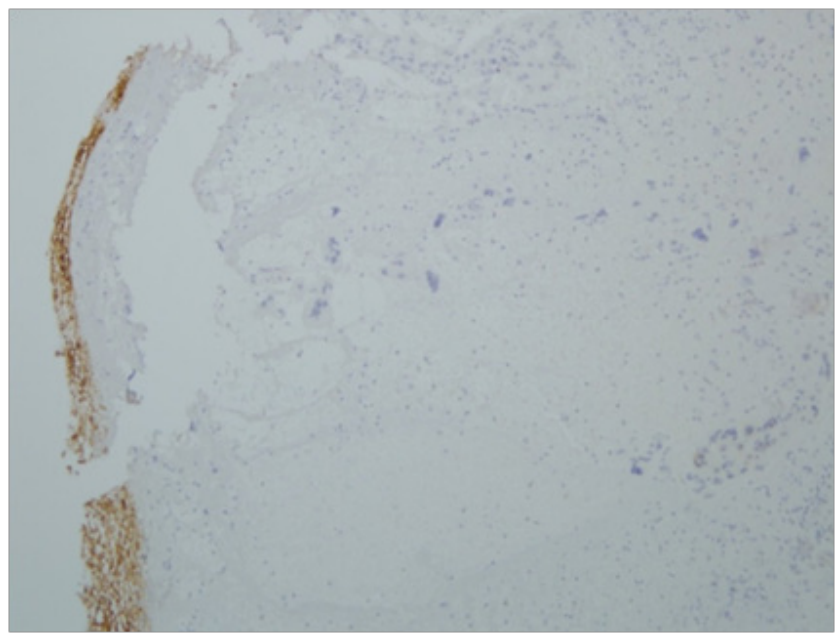

Figure 10 GFAP.

\section{Discussion}

Surgically resected blood clots from intracerebral hemorrhage (ICH) for histopathological examination are the frequent samples from neurosurgical department. Intracerebral hemorrhage has a number 
of causes, including: hypertension, head trauma, arteriovenous malformation, cavernoma, ruptured cerebral aneurysm, primary or metastatic neoplasm, bleeding disorder and medication. Incidence of bleeding is more in metastatic tumor than primary tumor. ${ }^{1}$ Most common metastatic tumors known to cause intracerebral hemorrhage are choriocarcinoma, bronchial carcinoma, melanoma and renal cell carcinoma. In our case, on H\&E examination, histopathologic differentials were metastatic choriocarcinoma, metastatic sarcoma and symplastic cavernoma/hemangioma. Cytokeratin positivity and negative vimentin ruled out possibility of sarcoma, negative CD31 ruled out hemangioma and positive HCG (with positive cytokeratin) confirmed metastatic choriocarcinoma. ${ }^{4}$ Serum/CSF beta-HCG ratio will be decreased in metastatic intracerebral choriocarcinoma, ${ }^{5}$ In this case, Serum/CSF beta-HCG ratio and other investigations could not be done as it was an unsuspected case transferred from outside hospital in critical condition and the patient went back to her own country for further management. The choriocarcinoma is a rare highly malignant vascular trophoblastic tumor with high incidence of metastasis. The most common sites for metastatic choriocarcinoma are lung, brain and liver. Rarely, it can metastasize to gastrointestinal tract, kidney, spleen, genital tract and lymph nodes.

\section{Conclusion}

Careful histopathologic examination of surgically removed intracerebral blood clot is very important and metastatic choriocarcinoma should be considered in differential diagnosis of intracerabral hemorrhage when a patient is a female in the reproductive age group. Though the metastatic cerebral choriocarcinoma is very rare, from the experience of this case, we recommend that serum and CSF beta-HCG should be done in all young female patients with intracerebral hemorrhage.

\section{Acknowledgements}

We would like to thank Dr Shatish Krihnan, HOD, Neurosurgical Department, Al Qassimi Hsopital (AQH) and all members of his team for their continuous support to our department. We would also like to thank all the three histotechnologists of our department for their hard work, interest in the subject and efficient performance to produce excellent quality of histopathology slides.

\section{Conflict of interest}

Both the authors have declared no conflict of interest for this work.

\section{References}

1. Suresh TN, Santosh V, Shastry Kolluri VR, et al. Intracranial haemorrhage resulting from unsuspected choriocarcinoma metastasis. Neurol India. 2001;49(3):231-236.

2. Milenković V1, Lazović B, Mirković L, et al. Brain metastases of choriocarcinoma-A report on two cases. Vojnosanit Pregl. 2013;70(10):968-971.

3. Shrestha P, Devkota U, Panth R. Metastatic Choriocarcinoma Masquerading As An Intracerebral Arteriovenous Malformation Bleed. The Internet Journal of Neurosurgery. 2009;7(2):1-4.

4. Kidd D, Plant GT, Scaravilli F, et al. Metastatic choriocarcinoma presenting as multiple intracerebral haemorrhages: the role of imaging in the elucidation of the pathology. J Neurol Neurosurg Psychiatry. 1998;5(6):939-941.

5. Rocque BG, Başkay MK. Spontaneous acute subdural hematoma as an initial presentation of choriocarcinoma: A case report. $J$ Med Case Rep. 2008;2:211. 